\title{
Using timing-based side channels for anomaly detection in industrial control systems
}

\author{
Stephen Dunlap, ${ }^{a}$ Jonathan Butts, ${ }^{b}$ Juan Lopez, ${ }^{c}$ Mason Rice, ${ }^{a 1}$ Barry \\ Mullins $^{a}$ \\ ${ }^{a}$ Department of Electrical and Computer Engineering, Air Force Institute of Technology, \\ Wright-Patterson Air Force Base, Ohio 45433, USA \\ ${ }^{b}$ QED Secure Solutions, 417 Forest Ridge Drive, Coppell, Texas 75019, USA \\ ${ }^{c}$ Applied Research Solutions, 51 Plum Street, Beavercreek, Ohio 45440, USA
}

\begin{abstract}
The critical infrastructure, which includes the electric power grid, railroads and water treatment facilities, is dependent on the proper operation of industrial control systems. However, malware such as Stuxnet has demonstrated the ability to alter industrial control system parameters to create physical effects. Of particular concern is malware that targets embedded devices that monitor and control system functionality, while masking the actions from plant operators and security analysts. Indeed, system security relies on guarantees that the assurance of these devices can be maintained throughout their lifetimes. This paper presents a novel approach that uses timing-based side channel analysis to establish a unique device fingerprint that helps detect unauthorized modifications of the device. The approach is applied to an Allen Bradley ControlLogix programmable logic controller where execution time measurements are collected and analyzed by a custom anomaly detection system to detect abnormal behavior. The anomaly detection system achieves true positive rates of 0.978 to 1.000 with false positive rates of 0.033 to 0.044 . The test results demonstrate the feasibility of using timing-based side channel analysis to detect anomalous behavior in programmable logic controllers.
\end{abstract}

\section{Keywords}

\footnotetext{
${ }^{1}$ Corresponding author: Mason Rice (mason.rice@afit.edu)
} 
Industrial Control Systems; Programmable Logic Controllers; Modification Attacks; Side Channels; Anomaly Detection

\section{Submitted: January 4, 2016 \\ Revision: April 3, 2016 \\ Accepted: July 21, 2016}

\section{Introduction}

During a siege in 590 BCE, Solon of Athens poisoned the water supply of the town of Cirrha using hellebore roots [22]. The contaminated water incapacitated the unsuspecting Cirrhaeans with uncontrollable diarrhea and the Athenians quickly overwhelmed the city [22]. In 2000, Vitek Boden leveraged unauthorized wireless access to a sewage treatment plant to release 800,000 liters of raw sewage into public water supplies in Australia [24]. In this incident, one malicious actor without direct physical access was able to cause a significant environmental impact. Due to increasing network connectivity, critical infrastructure systems are more susceptible to malicious attacks than ever before.

Modern society relies on industrial control systems (ICSs) to automate the operation of the critical infrastructure. Historically, industrial control systems were considered to be secure due to their isolation from external networks. Recently, however, industrial control systems have become less isolated as they incorporate commodity information technologies to improve efficiency and decrease costs [27]. The trend to interconnect industrial control devices exposes them to external networks and potential threats. Common devices, such as programmable logic controllers (PLCs), often lack security mechanisms such as authentication and encryption [27]. Stuxnet [8] demonstrated that significant damage can occur if a programmable logic controller in an industrial facility is compromised. Stuxnet also demonstrated that network protection mechanisms such as intrusion detection and air gapping, while important and effective, are unable to protect sensitive systems from sophisticated attackers.

A programmable logic controller has three layers: (i) hardware layer; (ii) firmware layer; and (iii) programming layer [4]. Building and maintaining trust in a device requires validating all three layers against a known-good 
baseline. The hardware is the lowest layer that executes the firmware. The firmware handles functionality such as communications, programming layer execution and error handling. Compromising the firmware of a device enables an attacker to cause negative effects and hide them from operators. The programming layer, also called the application layer, is designed to perform high-level system tasks. The applications in this programmable logic controller layer are often implemented using ladder logic programs. Stuxnet [8] demonstrated that the application layer of a programmable logic controller can be modified to cause significant damage to the controlled physical systems.

\section{Background}

This paper describes a novel technique for fingerprinting a programmable logic controller after it has been deployed. The fingerprint is developed by monitoring the execution characteristics of the device while it is in operation. The device is then fingerprinted periodically to verify that it has not been modified intentionally or unintentionally. The timing characteristics of the programmable logic controller are determined by the firmware and ladder logic programs that execute on the device. A baseline is created based on these characteristics and deviations from the baseline are flagged as anomalies.

This research employed an Allen Bradley ControlLogix L61 CPU module. Allen Bradley is a brand of Rockwell Automation, the second largest global supplier of programmable logic controllers [30]. The ControlLogix series of programmable logic controllers includes a task monitor utility capable of remotely collecting CPU execution times. This utility was designed to enable engineers to determine the CPU resources used by the firmware and ladder logic programs. Execution times are reported in microseconds and segmented into individual user tasks and firmware services such as communications. The ControlLogix utility provides sufficiently accurate timing information with minimal invasiveness. Alternative methods of collecting timing characteristics must considered for other programmable logic controllers.

Note that the effect of conditionally executed subroutines was not explored in this study. Subroutines in a ladder logic program may be designed to execute when certain conditions are met. These conditions and subroutine size can vary drastically between applications. Future research will attempt 
to characterize the possible effects and determine if the technique should be improved to handle these issues.

\subsection{Programmable logic controller security}

Most industrial control system security efforts have focused on the traditional information technology aspects of control systems [19, 27]. The information technology level refers to devices such as computers and firewalls, but it specifically excludes control system components and embedded devices such as programmable logic controllers. Cyber attacks on control systems are expected to increase in the future. The threats posed by cyber attacks are heightened by vulnerabilities in systems that support the critical infrastructure [29]. It is expected that adversaries will target the most vulnerable components in industrial control systems. Protecting the traditional information technology components of industrial control systems is necessary, albeit not sufficient, to secure critical infrastructure systems.

Programmable logic controllers are a major focus of industrial control system security efforts. A programmable logic controller is often a fragile device that is prone to failure from cyber-induced events, especially one that presents unexpected inputs such as malformed packets to the device [27]. Programmable logic controllers are designed to be reliable and easy to manage under normal conditions; unfortunately, most devices are not designed with security as a primary requirement. As such, simple security features such as authentication and encryption are often not implemented. Without these protection mechanisms, programmable logic controllers are highly vulnerable targets. Firmware, ladder logic programs and device configurations are also susceptible to manipulation by an adversary intent on disrupting control system operations [15].

Ensuring that a programmable logic controller has not been maliciously modified after its deployment is a major challenge. The device must be analyzed comprehensively to demonstrate that it can be trusted. This requires the validation of the hardware, firmware, ladder logic programs and configuration settings. Firmware validation is an important first step toward establishing trust [4]. After a device has been shown to be trustworthy, however, the trust must be re-validated at periodic intervals. Verifying that a device has not been modified is the most direct means of maintaining trust. Firewalls and intrusion detection systems are important, but they are not enough [3]. A general method for confirming the correct operation of a device must be developed to ensure that it has not been modified in a malicious 
manner.

\subsection{Side channel analysis}

A side channel is an unintended avenue by which information can be obtained by an observer; this is also referred to as an information leak [11]. Side channel information is typically leaked due to the physical requirements of devices [1]. Monitoring the physical aspects of a device can provide information about its internal state and operation [31]. A side channel analysis attack occurs when an adversary uses leaked information to learn secrets contained within a system $[1,6,11]$. The targets of these attacks are typically encryption keys [1, 5, 7, 13, 14], PIN numbers [7], passwords [25] and user data [25]. The most common side channel analysis attack methods are power analysis, electromagnetic analysis and timing analysis $[2,16,31]$.

\subsubsection{Timing analysis}

This research focuses on the use of timing data to obtain information about the operation of a programmable logic controller. Timing attacks have been used in the past to attack cryptosystems to recover secret keys [1, $5,7,13]$; they have also been used against systems to obtain other secret information (e.g., user data [25]). Any algorithm that does not execute in constant time potentially leaks information. Zhou and Feng [31] list three conditions necessary to discover information. First, the execution time must be at least partially dependent on a secret within the system. Second, the attacker must be able to collect enough time measurements to statistically analyze the data. Third, the attacker must be able to measure the time with known or consistent error.

Kocher [13] presented the idea of timing-based side channel analysis in 1996. The experiment involved an eavesdropper listening to an interactive protocol in which one computer received requests in plaintext and returned RSA-encrypted ciphertext. When the encryption system received a request, the amount of time taken by the system to respond was measured. The execution time partially depended on the secret key because the algorithm used for modular exponentiation skipped a modular multiplication operation for each bit of the key that was set to zero. To compensate for the noise introduced by random or uncontrollable factors, multiple measurements were taken to obtain enough data for statistical analysis. The first bit of the key was guessed, the encryption was simulated and the time taken to complete 
each message based on measurements taken on the same system was estimated. Upon subtracting the simulated values from the measured values, the total variance was observed to decrease if the guess of the first bit was correct; if the guess was incorrect, then the variance was observed to increase. This process was repeated for each bit of the key, allowing the recovery of the RSA key one bit at a time. This approach, however, required in-depth knowledge of the encryption system. Experiments were also conducted to obtain information about the time required to complete specific portions of the encryption algorithm. This made the attack more theoretical than practical because the amount of information required could be prohibitively high.

Dhem et al. [7] built on Kocher's work to bypass encryption mechanisms in CASCADE smart cards that used Montgomery multiplication to optimize the encryption. Schindler [23] introduced a new timing attack against RSA implementations that used Montgomery multiplication and the Chinese remainder theorem. Brumley and Boneh [5] demonstrated the practicality of remote timing attacks on a more powerful machine - this attack vector was generally not believed possible due to the multitasking operating systems employed at the time. More recently, Aciicmez et al. [1] performed a remote timing cache attack that demonstrated the power of timing data with a sufficient number of measurements.

\subsubsection{Side channel analysis for anomaly detection}

In 2012, Stone et al. [26] demonstrated the use of side channel analysis to detect anomalous behavior in programmable logic controllers. They used a near-field RF probe to collect emanations from a programmable logic controller and obtained a fingerprint of normal device operation. This baseline fingerprint was used to detect deviations from normal operation. Devices were classified via 1-to-n comparisons using the fingerprint of one device to validate the operation of suspect devices. Stone et al. also demonstrated the ability to authenticate devices using 1-to-1 comparisons. This technique enabled a device to be fingerprinted once and authenticated in the future using the fingerprint. Stone et al. showed that clear differences existed between the signals collected from the normal device and the signals collected from the anomalous device.

Although the technique presented by Stone et al. is promising, it requires additional hardware to be added to a programmable logic controller - this makes the approach invasive and expensive. No other research applying side channel analysis for anomaly detection has been identified. Thus, the pro- 


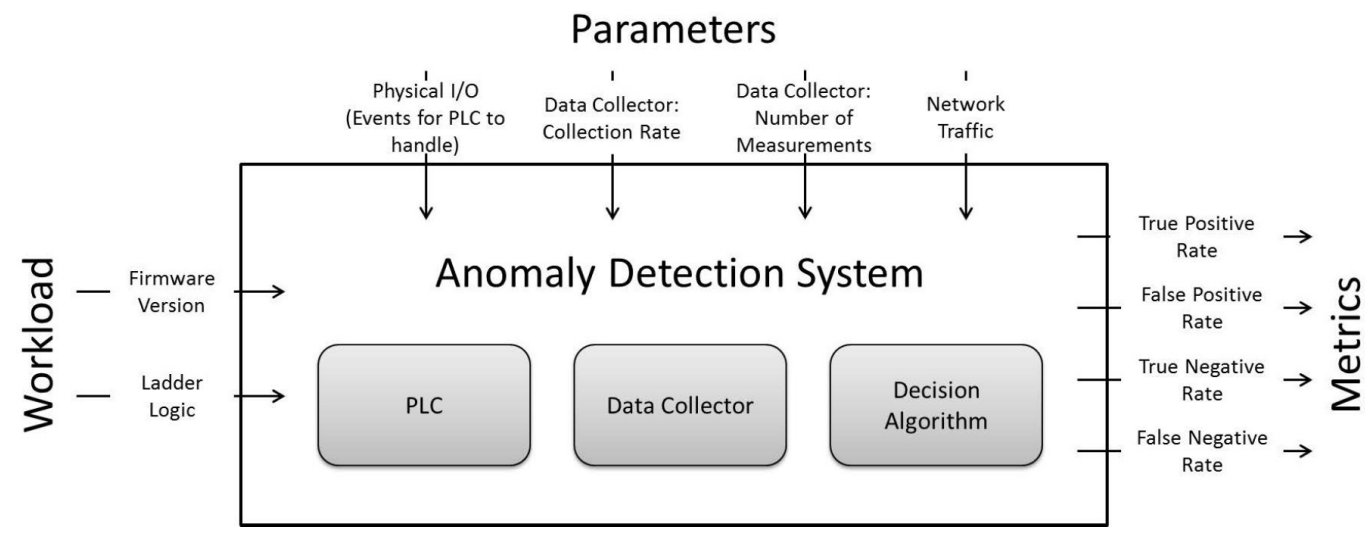

Figure 1: Anomaly detection system.

posed technique that applies timing-based side channel analysis for anomaly detection in programmable logic controllers is, indeed, novel. In fact, the technique can help detect modifications to the firmware and ladder logic programs in programmable logic controllers.

\section{Anomaly detection system}

This section describes the anomaly detection system that uses side channel analysis to detect unauthorized modifications made to programmable logic controller firmware and ladder logic programs. The approach analyzes the execution time characteristics of programmable logic controllers to detect intentional or unintentional modifications.

\subsection{System description}

Figure 1 shows a schematic diagram of the overall system, which consists of a programmable logic controller, data collector and decision algorithm. Firmware and ladder logic programs are loaded in the programmable logic controller and baseline timing data is collected during its initial operation. Timing data is collected during subsequent operation of the programmable logic controller. An anomaly is deemed to exist if the decision algorithm determines that differences exist between the two sets of timing data. An Allen Bradley ControlLogix programmable logic controller was used in this study because of its ubiquity in industrial facilities and its remote task monitoring feature. 


\subsection{Workload}

The workload used for anomaly detection consisted of firmware versions and ladder logic programs. In the experiments, different firmware versions were loaded in the programmable logic controller to determine if the anomaly detection system could distinguish between the firmware versions. The ability to detect different firmware versions is indicative of the ability to detect modified firmware.

Two ladder logic programs were also introduced into the programmable logic controller. The number and type of operations in a ladder logic program affects the time required for the processor to execute one scan of the program. Modifications made to a large ladder logic program should be more difficult to detect than modifications made to a small program. The two programs were developed to evaluate this expectation. One program was written to exercise as much of the programmable logic controller functionality as possible; the other was written to accomplish a very small task. These two extremes provide information about the impact of ladder logic program complexity on anomaly detection.

\subsection{Performance metrics}

The anomaly detection system compares two datasets, one corresponding to the baseline and the other collected during programmable logic controller operation. The anomaly detection system reports one of two outcomes: (i) an anomaly exists, when the two datasets are determined to be different; or (ii) no anomaly exists, when the two datasets are determined not to be different. Each outcome is classified as follows:

- True positive (TP): An anomaly exists and is detected.

- False positive (FP): An anomaly does not exist but is detected.

- True negative (TN): An anomaly does not exist and is not detected.

- False negative (FN): An anomaly exists but is not detected.

Common measures of accuracy for diagnostic systems are the true positive rate (TPR) and false positive rate (FPR) [28]. These metrics are used to evaluate the effectiveness of a system at identifying individual anomalies. The true positive rate, which corresponds to the number of correctly identified anomalies per total number of anomalies, is given by: 


$$
T P R=\frac{T P}{T P+F N}
$$

The false positive rate, which corresponds to the number of incorrectly identified anomalies per total number of instances where an anomaly does not exist, is given by:

$$
F P R=\frac{F P}{F P+T N}
$$

The false negative rate (FNR) and true negative rate (TNR) are defined in terms of the true positive rate and false positive rate, respectively:

$$
\begin{aligned}
& F N R=1-T P R \\
& T N R=1-F P R
\end{aligned}
$$

Note that the false negative rate and true negative rate metrics are implicitly represented in the true positive rate and false positive rate metrics. In fact, Equations (3) and (4) are presented for completeness. The true positive rate and false positive rate metrics are used to evaluate the anomaly detection results.

Acceptable values of the true positive and false positive rates depend on the application. In this study, a true positive rate greater than 0.9 (i.e., 90\%) and a false positive rate less than $0.1(10 \%)$ were deemed to be acceptable.

\subsection{System parameters}

This section describes the parameters that affect the performance of the anomaly detection system.

- Analog and digital inputs and outputs: Changes to physical inputs must be processed by the programmable logic controller. An increase in the frequency of changes is expected to correlate to an increase in variation in the execution times. Pilot studies indicated that the effects on the device were small and that the specific effects were outside the scope of this study. Therefore, no physical inputs or outputs were considered in this study.

- Collection rate: The rate of data collection may also affect the performance of the programmable logic controller. Since the execution 
times were collected over the network, the additional communications had to be handled by the programmable logic controller. Pilot studies were performed to evaluate this effect, which was found to be minimal. A rate of four measurements per second was selected for this study.

- Number of measurements: The number of execution time measurements is expected to affect the accuracy of the decision algorithm. The accuracy of the algorithm should improve given more measurements. The number of measurements also affects the utility of the system. The amount of time needed to make a decision is determined by the collection rate and the number of measurements. A pilot study revealed that collecting 10,000 measurements for each dataset provided adequate data for the decision algorithm to make a determination. At a collection rate of four measurements per second, 10,000 measurements can be collected in approximately 42 minutes. 10,000 measurements were collected for each experiment, but subsets of the data were also analyzed to determine the minimum number of measurements needed to achieve acceptable accuracy.

- Network traffic: Network traffic must be handled by the programmable logic controller. The network traffic in the experiments was limited to traffic generated for data collection.

\subsection{Ladder logic applications}

This study used two ladder logic programs. A modification to a small ladder logic program should be easier to detect than a modification to a large ladder logic program. The two programs were designed to evaluate this expectation. The mean execution time for Program 1 was less than $110 \mu s$. The mean execution time for Program 2 was greater than 3,000 $\mu s$.

- Program 1: Program 1 was derived from a sample program provided with the Allen Bradley RSLogix 5000 software. This temperature control program involves the three tasks shown in Figure 2: (i) Loop_1_Sec; (ii) InputControl; and (iii) MainTask. Loop_1_Sec contains a PID (proportional integral derivative) control object and is configured for periodic execution every second. The PID control takes an analog input from a temperature sensor and controls an analog output to a heating element. In this study, the analog input was replaced by a simulated input controlled by InputControl. To enable the PID control to reach 


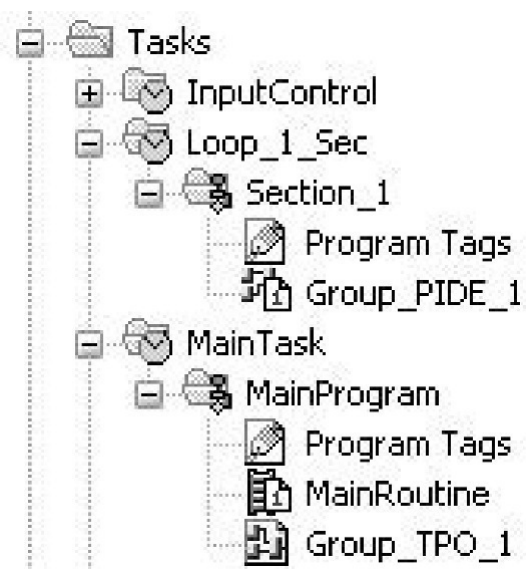

Figure 2: Temperature control program.

steady state quickly, the input value was set to be equal to the set point value when the programmable logic controller was first initialized. The simulated input provides work for the PID control without having to use a physical system. InputControl modifies the input value based on the output value of the PID control. MainTask, shown in Figure 3, controls the tuning capabilities of the PID control object. MainTask is an ideal target for an adversary because it can readily modify the PID control. It is also relatively complex and small modifications could go unnoticed when examined by an control engineer. MainTask was configured for periodic execution every $250 \mathrm{~ms}$ and the mean execution time was less than $110 \mu \mathrm{s}$ for all the firmware versions considered.

- Program 2: Program 2 was specifically written for this research. It had a mean execution time greater than 3,000 $\mu s$ for all the firmware versions considered. The program was developed to determine the impacts of ladder logic program size and complexity on anomaly detection. The program incorporated 32 instructions that were repeated 115 times.

The four basic categories of operations selected were integer operations, floating point operations, Boolean operations, timers and counters. While other categories of operations are available, the selected categories represent the basic operations associated with ladder logic programs. 


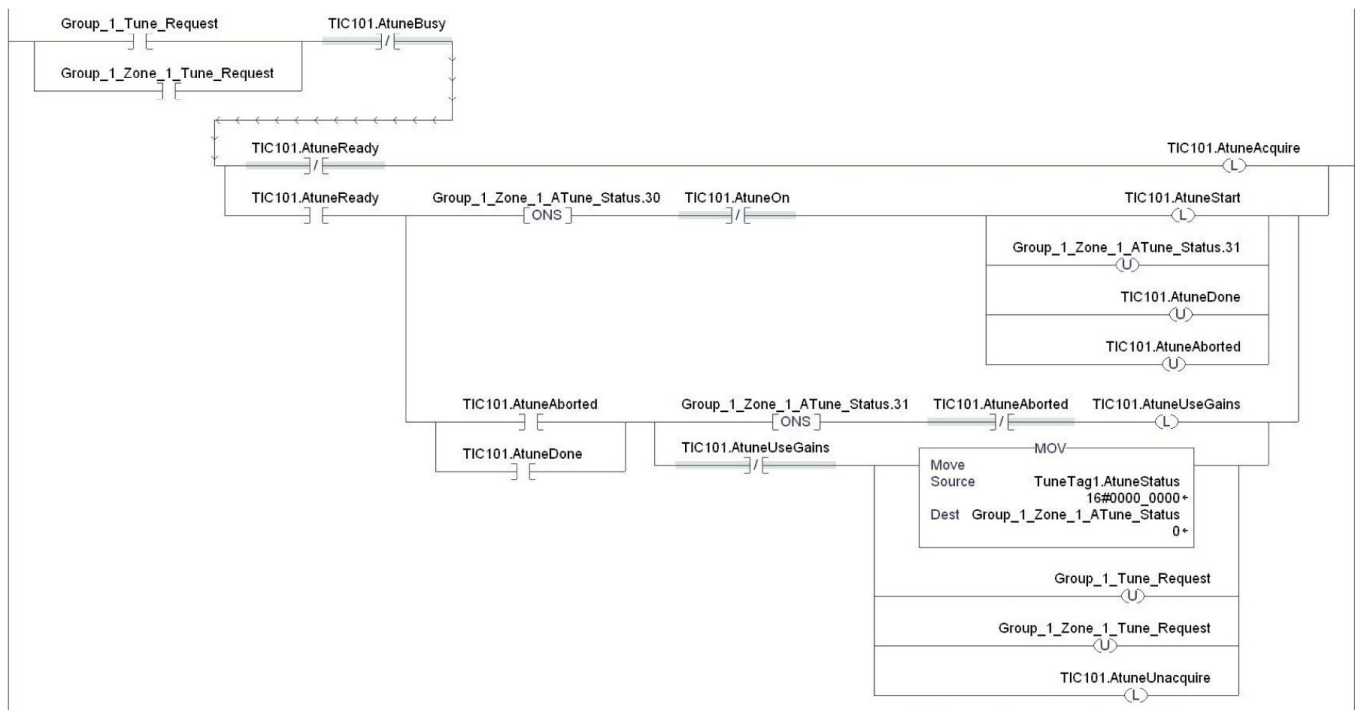

Figure 3: Temperature control program (MainRoutine).

Table 1: Factors and levels.

\begin{tabular}{llll}
\hline & Level 1 & Level 2 & Level 3 \\
\hline Firmware & V 16.056.47 & V 19.011.16 & V 19.015.25 \\
Ladder Logic & No Modification & Simple Modification & Complex Modification \\
\hline
\end{tabular}

Ladder logic programs receive inputs from external sources and physical processes. The program inputs change over time; a program with static inputs is not realistic. To account for the variations caused by changing inputs, Program 2 was made more complex by randomizing the inputs to the operations at the beginning of each run. A pseudorandom number generator was implemented in ladder logic and was seeded with the system time during initialization. The random number generator was derived from an example program in [12] and was included in a separate task. The values produced by this task were used to set the input variables at the beginning of the main task.

\subsection{Firmware}

Table 1 shows the programmable logic controller firmware versions used in the study. Comparing different firmware versions with identical ladder logic 
programs can reveal the differences in execution times caused by modifying the firmware. Because malicious firmware samples were not available, different firmware revisions from Allen Bradley were employed. The following firmware versions were used in the study:

- Version 16.056.47: Version 16.056.47 corresponds to a major firmware revision. A major revision is expected to have more modifications than a minor revision. These modifications include new features and optimizations. The effect of a major revision change was evaluated in the study.

- Versions 19.011.16 and 19.015.25: Versions 19.011.16 and 19.015.25 correspond to minor revisions within the same major revision. A minor revision change is expected to have fewer modifications than a major revision and is expected to be more difficult to detect.

\subsection{Ladder logic program modifications}

Comparing different ladder logic programs with the same firmware version reveals differences in the execution times due to the additional ladder logic operations. Three ladder logic program modifications were used in the study:

- No modification: The unmodified ladder logic program provided a baseline fingerprint for comparing the effects of added operations.

- Simple modification: The simple modification was designed to cause one branch of a ladder logic program to always evaluate to false. Pilot studies revealed that ladder logic operations are evaluated by the programmable logic controller regardless of the current value of the rung. Thus, the execution time should increase due to the additional operation. This simple modification should be more difficult to detect than a complex modification.

Program 1 incorporated an Examine if Closed operation to disable the triggering of an auto tuning routine (Figure 4). Similarly, Program 2 incorporated an Examine if Open operation to cause one rung to always evaluate to false, preventing one "Unlatch" operation from occurring.

- Complex modification: The complex modification added one rung with an integer multiplication operation to each ladder logic program. 


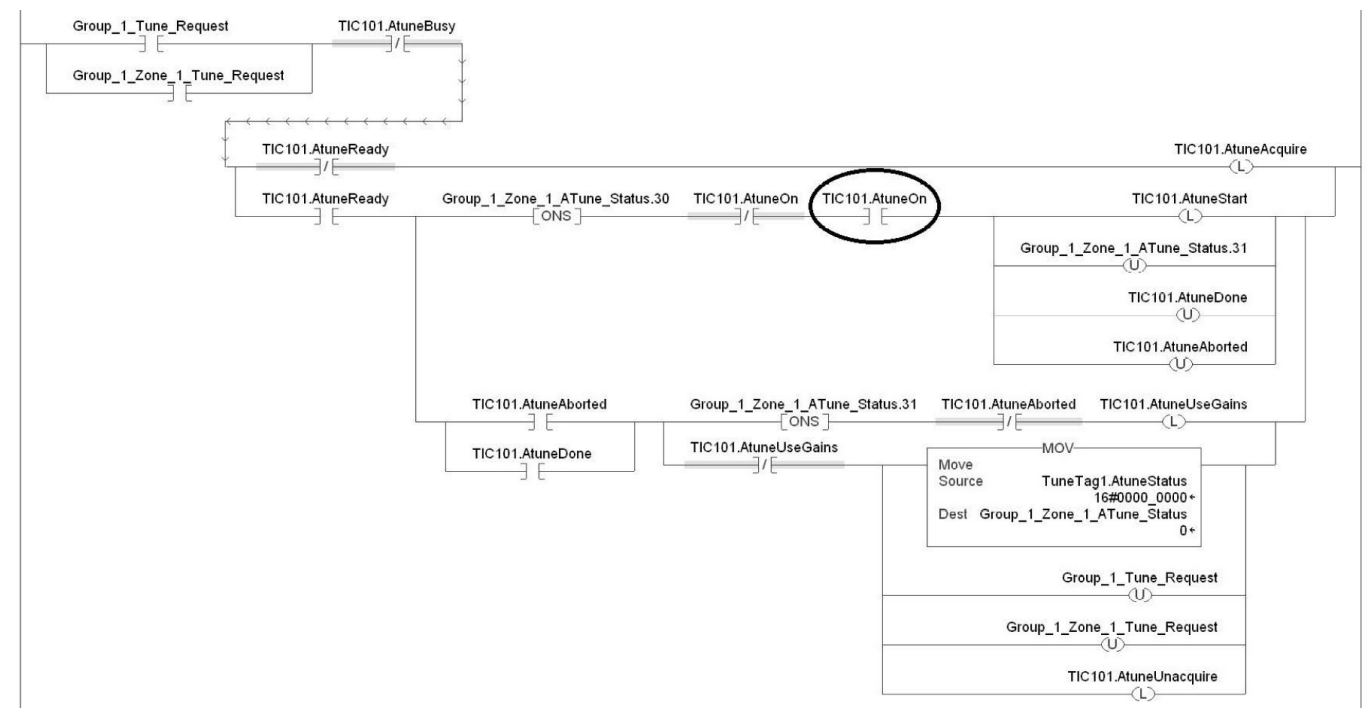

Figure 4: Temperature control program (MainRoutine with a simple modification).

An integer multiplication operation is more complex and computationally expensive than the Boolean operation used in the simple modification. Specifically, an integer variable is multiplied by a constant value and the result is stored in a variable that is not referenced elsewhere in the program. The multiplication operation had no effect on the program logic.

\section{Experimental design}

The test equipment for the experiments included an Allen Bradley ControlLogix programmable logic controller with a 1756-L61 CPU module and a Windows XP computer with RSLogix 5000 and Python installed. The two devices were connected via Ethernet to a common switch.

ControlLogix series programmable logic controllers have a feature that allows users to obtain performance data over Ethernet. This feature was important because it enabled remote characterization with minimal intrusiveness. The performance data was segmented by task and included the last execution time in microseconds. The execution times of the ladder logic programs were collected from the programmable logic controller and were used to detect anomalous behavior. 
A full factorial design was employed for the experiments. Each repetition consisted of nine unique experiments for Program 1 and nine unique experiments for Program 2 (18 total). To ensure sufficient data for comparative analysis, five replications were used for each experiment, yielding 45 individual experiments per application (90 total).

Based on the experimental results, a receiver operating characteristic (ROC) curve was plotted to determine the decision threshold that yielded the highest true positive rate with the lowest false positive rate. According to Japkowicz and Shah [10], a ROC curve of a classifier is a plot where the $\mathrm{y}$-axis represents its true positive rate and the $\mathrm{x}$-axis represents its false positive rate. The true positive and false positive rates were generated by varying the decision threshold. In this study, the classifier was the anomaly detection system and the decision threshold determined whether or not a difference between two experiments was anomalous. The area under the ROC curve is often used as a performance metric for classification systems. The maximum possible area under the ROC curve is one. If the area under the ROC curve is equal to one, perfect discrimination is indicated [28]. In practice, a decision threshold is selected by evaluating the potential damage caused by a false negative error and the cost incurred by a false positive error.

Every step of the experiments was automated to reduce human error. Each experiment involved the following procedure:

1. Firmware was installed in the programmable logic controller.

2. A ladder logic program was installed in the programmable logic controller.

3. The firmware version number was requested from the programmable logic controller.

4. The ladder logic program name was requested from the programmable logic controller.

5. The firmware version number and ladder logic program name were verified against the desired values.

6. Ten thousand measurements were obtained from the data collector.

The Allen Bradley ControlFlash application is often used to install firmware on ControlLogix programmable logic controllers. However, this application did not meet the automation requirements of the research. To enable the configuration and execution of experiments, a custom application was developed to install firmware in the programmable logic controller. The custom application incorporated the same communications routine as ControlFlash and 
provided equivalent functionality. At the beginning of each experiment, the desired firmware file was installed on the programmable logic controller and the device was restarted. The current firmware revision was requested from the programmable logic controller and verified against the desired firmware version.

The Microsoft UI Automation Library and C\# were used to automate the RSLogix 5000 software. RSLogix 5000 is a software package provided by Rockwell Automation for programming and configuring Allen Bradley programmable logic controllers. The UI Automation Library was used to control RSLogix 5000 by clicking on the buttons needed to program the programmable logic controller. Each ladder logic program was given a unique project name. After programming the programmable logic controller, the program name was requested from the programmable logic controller and the value was validated.

The data collector waited for 60 seconds before starting data collection. This enabled the programmable logic controller to reach a steady state of operation before the collection began. During the data collection, the application initiated a request for the CPU execution time every $250 \mathrm{~ms}$. The $250 \mathrm{~ms}$ time was based on the Windows XP system time and was not affected by delays during communications; it also prevented inconsistent communications delays from influencing the timing of the measurements.

After 10,000 measurements were collected, the data was stored and the next experiment was initiated. The device was reset to the initial state during the firmware flashing process at the beginning of each experiment. Note that the execution order of the experiments was randomized using the List Randomizer from random. org. The randomization reduced the influence of environmental factors that were not accounted for in the research.

\section{Implementation and results}

This section provides details of the anomaly detection system implementation and the anomaly detection results.

\subsection{Data collection}

To facilitate the research, the communications routine used by the task monitor utility provided with RSLogix 5000 was reverse engineered and a custom Python application was written to collect timing measurements using 
Table 2: EtherNet/IP header format.

\begin{tabular}{|l|c|c|c|c|c|c|c|}
\hline $\mathbf{1}$ & $\mathbf{2}$ & $\mathbf{3}$ & $\mathbf{4}$ & $\mathbf{5}$ & $\mathbf{6}$ & $\mathbf{7}$ & $\mathbf{8}$ \\
\hline Command & Data Length & \multicolumn{4}{|c|}{ Session Handle } \\
\hline \multicolumn{3}{|c|}{ Status } & \multicolumn{4}{c|}{ Sender Context... } \\
\hline \multicolumn{3}{|c|}{.Sender Context (continued) } & \multicolumn{4}{c|}{ Options } \\
\hline
\end{tabular}

the routine. The custom application was designed to automate data collection and provide precise control over the timing of the measurements. The task monitor utility used EtherNet/IP and the Common Industrial Protocol (CIP) to request execution times from the programmable logic controller.

\subsubsection{EtherNet/IP}

EtherNet/IP is a carrier protocol that standardizes communications across multiple media, including RS-232, RS-485 and Ethernet. It provides session handling, error detection and delivery confirmation. EtherNet/IP messages consist of a 24-byte header and a command-specific section. Each field in EtherNet/IP is in the little endian format.

Table 2 shows the EtherNet/IP header structure. The first field of the EtherNet/IP header is a two-byte command field. The next two bytes are the length (in bytes) of the command-specific portion of the packet. The next four bytes are the session handle, followed by a four-byte status field. The next eight bytes are the sender context, followed by a four-byte options field.

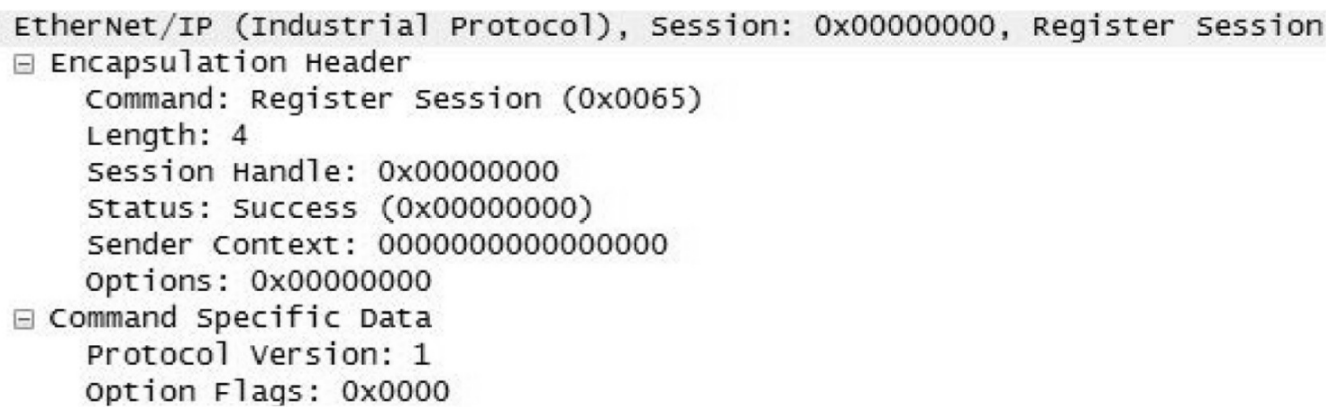

Figure 5: EtherNet/IP register session.

Before CIP requests can be made, a session handle must be requested from the programmable logic controller. Figure 5 shows a Wireshark rep- 
Table 3: EtherNet/IP payload format.

\begin{tabular}{|c|c|c|c|c|c|c|c|}
\hline $\mathbf{1}$ & $\mathbf{2}$ & $\mathbf{3}$ & $\mathbf{4}$ & $\mathbf{5}$ & $\mathbf{6}$ & $\mathbf{7}$ & $\mathbf{8}$ \\
\hline \multicolumn{3}{|c|}{ Interface Handle } & \multicolumn{2}{|c|}{ Timeout } & Item Count \\
\hline Item Type ID & Length & \multicolumn{3}{|c|}{ Item Data (Length Bytes) } \\
\hline Item Type ID & Length & \multicolumn{3}{|c|}{ Item Data (Length Bytes) } \\
\hline \multicolumn{3}{|c}{$\ldots$} \\
\hline
\end{tabular}

resentation of the register session request. The register session command (0x0065) is used to request a session handle from the programmable logic controller. The length field in the request is set to 0x0004. All the other fields in the header are set to zero. The command-specific portion of the packet is 0x0001 for CIP version 1 and 0x0000 for the option flags. If successful, the response from the programmable logic controller contains the newly-created session handle in the session handle field. The rest of the response is identical to the request. CIP requests can be sent using the new session handle.

A CIP request must be wrapped in an EtherNet/IP header. A common command in Ethernet/IP messages is "Send Data" (0x006F), which simply sends data, such as a CIP message, to a target device. The length of the command-specific data depends on the specific CIP request. The commandspecific portion of the packet has six fields (shown in Table 3). The four-byte interface handle specifies the protocol of the payload; the handle for CIP is zero. The two-byte timeout field may be selected as desired; in the experiment, this field was set to 0x0005. The CIP message is sent as EtherNet/IP items. The first two bytes of the items section is the number of items to be sent. Each item consists of a two-byte type ID and a two-byte length value, followed by the actual data. CIP requests for ladder logic program execution times can be sent as an unconnected data item (type 0x00B2).

\subsubsection{Common Industrial Protocol}

CIP is an object-oriented protocol that provides a suite of messages and services for automation applications [18]. It provides a common application layer for Ethernet/IP, ControlNet and DeviceNet networks [17]. CIP requests comprise services, class objects, instance numbers, attributes and data. Some of these values are specified in standards, but most are defined by device manufacturers. CIP provides the capability to route commands to the various modules on the backplane of a ControlLogix programmable 


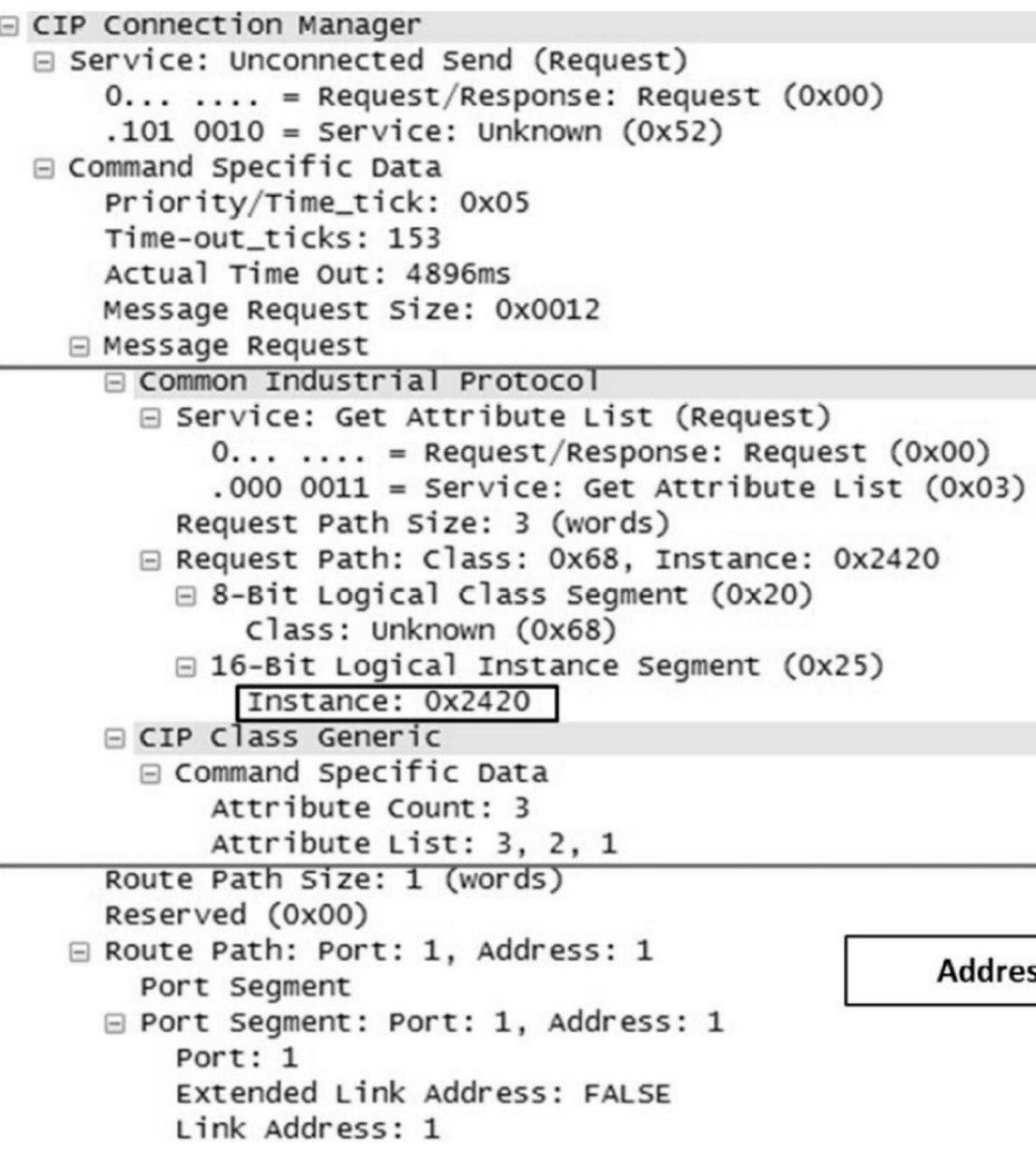

Figure 6: CIP request for ladder logic execution time.

logic controller. This is done using the standard connection manager object (class 6, instance 1). The object allows embedded CIP messages to be sent (using service 0x52 for example) across the backplane to other modules. The last parameter in this type of request is the address field. The address is the slot number of the target module on the backplane.

Figure 6 shows a request for the ladder logic program execution time from the ControlLogix L61 CPU module. The Connection Manager Request uses service 0x52 to send the Embedded CIP Request to slot 1 of the programmable logic controller that contained the L61 CPU module used in this study.

The Embedded CIP Request uses the Get Attribute List service (0x03) to 


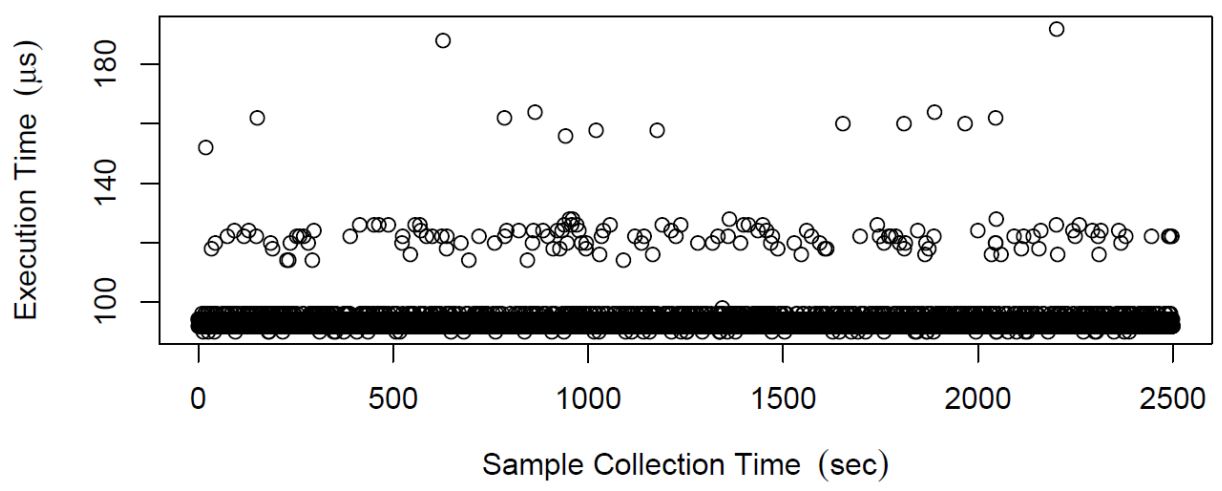

Figure 7: Program 1 execution times.

request the current values of attributes 1,2 and 3 for a given instance. Each user task is represented by an instance of class $0 x 68$. The instance number of the main task depends on the specific ladder logic program and firmware version. The task monitor utility attempts to request times by scanning a list of instances until it receives a successful response from the programmable logic controller. In this study, instead of scanning a list of instances, the correct instance number of each ladder logic program and firmware combination was recorded. Recording the instance numbers eliminated the overhead caused by invalid CIP requests.

\subsection{Collected data}

Ninety sets of 10,000 execution time measurements were collected. The time measurements are in microseconds with a resolution of two microseconds. The L61 CPU module uses interrupts to handle communications and scheduled events. When an interrupt occurs during ladder logic program execution, the amount of time that the processor spends responding to the interrupt is inadvertently added to the reported execution time. Consequently, techniques were applied to ensure that the data is representative of the ladder logic program execution times.

\subsubsection{Program 1}

Recall that Program 1 is a temperature control application with a mean execution time less than $110 \mu \mathrm{s}$. Figure 7 shows a representative dataset from an experiment using Program 1. In this dataset, measurements greater than $110 \mu s$ were caused by interrupts or other firmware functionality. 


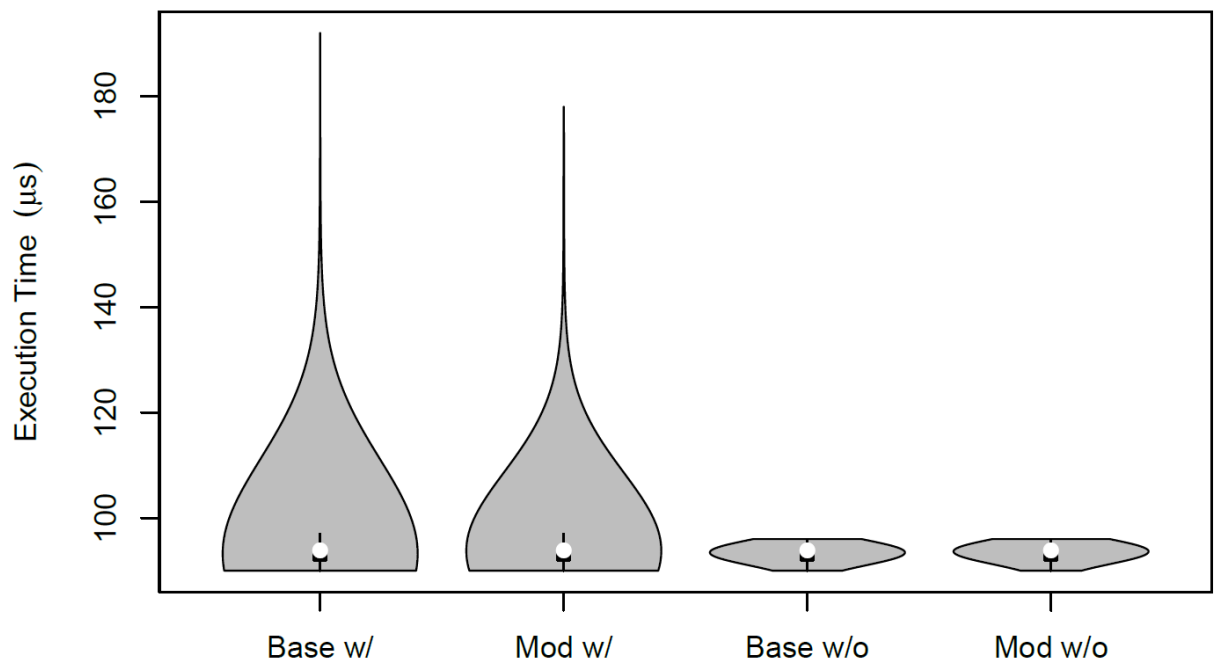

Figure 8: Example Program 1 comparison.

Figure 8 shows violin plots that compare two programmable logic controller configurations using firmware version 19.015. The two violin plots on the left include all the statistical outliers that comprise approximately $1 \%$ of the measurements. The difference between the two datasets is masked by the skewness of the data. The two violin plots on the right exclude statistical outliers. Although the range of execution times is roughly the same, further examination reveals that the distribution of values is higher with the introduced modification. Note that this difference is more apparent when the statistical outliers are removed. As such, the cluster with the lowest mean execution time is representative of the ladder logic program execution time and is the only data provided to the decision algorithm.

\subsubsection{Program 2}

Program 2 is a custom ladder logic program that uses a variety of operation types. Figure 9 shows a representative example dataset containing 10,000 measurements taken $250 \mathrm{~ms}$ apart. Note that, in the case of Program 1, the cluster with the lowest mean execution time comprises $99 \%$ of the data points, whereas the cluster with the lowest mean execution time for Program 2 comprises only $20 \%$ of the data points. As shown in Figure 9 , multiple distinct clusters are caused by interrupts and other unknown firmware functionality. As the average execution time of the ladder logic program increases, the probability of interrupts is expected to increase as 


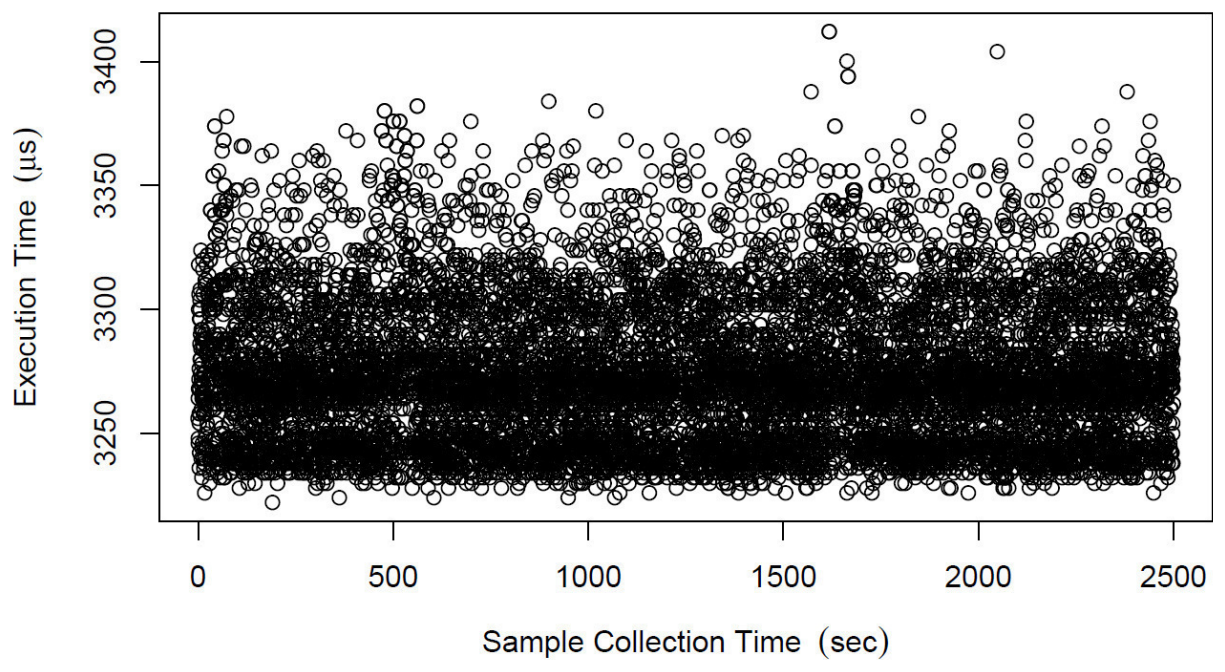

Figure 9: Program 2 execution times.

well.

Pilot studies revealed that the percentage steadily increases as the ladder logic program execution time increases. Figure 10 compares the results for two programmable logic controller configurations with firmware version 19.015. The two violin plots on the left include all the clusters. The difference between the two datasets is masked by the variations between the clusters. The two violin plots on the right exclude the upper clusters so that only the cluster with the lowest mean execution time is included. The difference in mean execution time is more apparent when only one cluster is taken into account. The cluster with the lowest mean execution time is indicative of ladder logic program execution without interrupts. To ensure that the data accurately reflected the execution times, only the lowest $20 \%$ of the measurements were provided to the decision algorithm.

\subsubsection{Program 1 execution characteristics}

This section discusses the execution characteristics of Program 1. Specifically, it presents the mean execution time for each ladder logic program and firmware combination and provides insights into the actual effects caused by the modifications. Table 4 presents the mean execution times for the five repetitions of Program 1. For each ladder logic program and firmware combination, the mean, standard deviation, minimum and maximum of the mean execution times for the five repetitions are presented. Note that $\mathrm{N}$ 


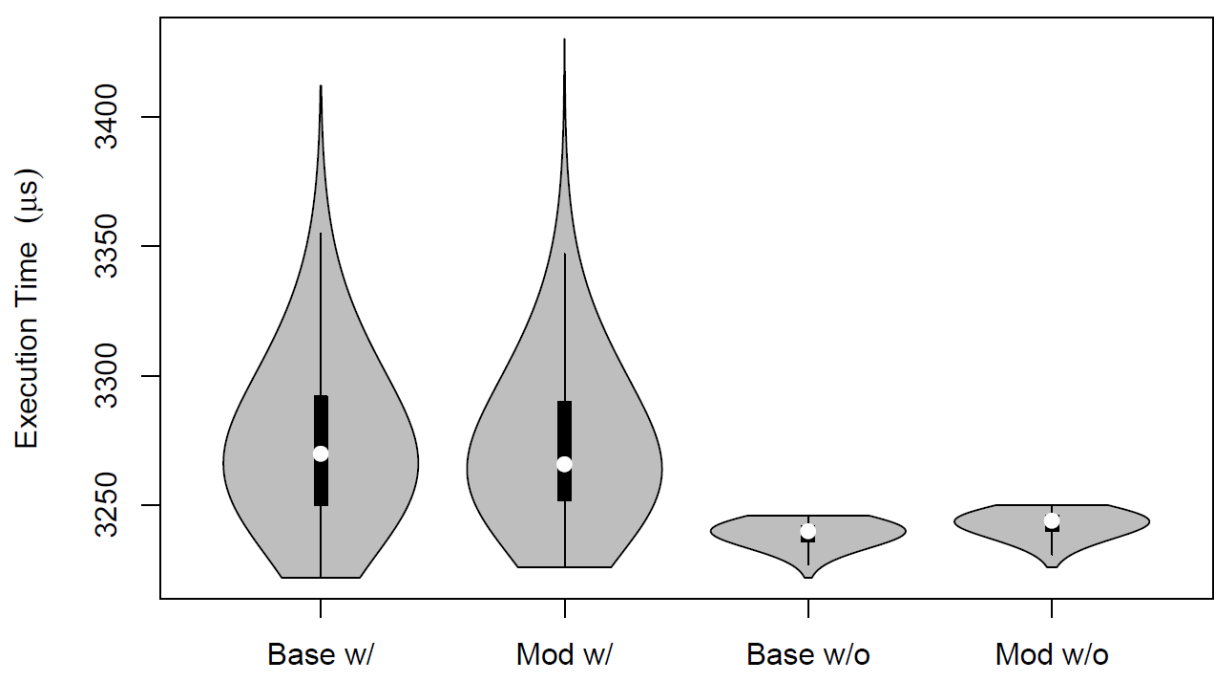

Figure 10: Example Program 2 comparison.

corresponds to no modification, $\mathrm{S}$ to simple modification and $\mathrm{C}$ to complex modification.

- Ladder logic program modification: For firmware version 16.056 with no modification to the ladder logic program (16.056-N), the mean execution time is $90.40 \mu \mathrm{s}$. With the simple modification (16.056-S), the mean execution time decreases to $90.28 \mu \mathrm{s}-$ a difference of $0.12 \mu \mathrm{s}$. Note that, since the standard deviation of $16.056-\mathrm{N}$ is $0.002 \mu \mathrm{s}$, the mean execution time of $16.056-\mathrm{S}$ is 60 standard deviations away from the mean execution time of $16.056-\mathrm{N}$. The $0.12 \mu \mathrm{s}$ difference is the smallest observed difference in mean execution times caused by a ladder logic program modification. For firmware version 16.056 with the complex modification (16.056-C), the mean execution time is $101.05 \mu \mathrm{s}$, which is 5,325 standard deviations away from the mean execution time of $16.056-\mathrm{N}$. The large differences in mean execution times show that the modifications are readily identifiable.

Similarly, for firmware versions 19.011 and 19.015, modifications to the ladder logic programs create significant differences in the mean execution times that are readily discernible. Figure 11 presents the mean execution times for Program 1. Note that, since the standard deviation for each dataset in Figure 11 is approximately $0.01 \mu \mathrm{s}$, the confidence intervals are too small to present in the graph. Nevertheless, 
Table 4: Execution times ( $\mu s)$ for Program 1 (five repetitions).

\begin{tabular}{|c|c|c|c|c|c|}
\cline { 3 - 6 } \multicolumn{2}{c|}{} & Mean & SD & Min & Max \\
\hline \multirow{3}{*}{ V 16.056 } & N & 90.40 & 0.002 & 90.40 & 90.41 \\
\cline { 2 - 6 } & $\mathbf{S}$ & 90.28 & 0.014 & 90.26 & 90.29 \\
\cline { 2 - 6 } & $\mathbf{C}$ & 101.05 & 0.008 & 101.04 & 101.06 \\
\hline \multirow{3}{*}{$\mathbf{1 9 . 0 1 1}$} & $\mathbf{N}$ & 93.61 & 0.007 & 93.61 & 93.62 \\
\cline { 2 - 6 } & $\mathbf{S}$ & 93.44 & 0.007 & 93.43 & 93.45 \\
\cline { 2 - 6 } & $\mathbf{C}$ & 103.67 & 0.012 & 103.66 & 103.69 \\
\hline \multirow{3}{*}{$\mathbf{1 9 . 0 1 5}$} & $\mathbf{N}$ & 93.42 & 0.015 & 93.40 & 93.44 \\
\cline { 2 - 6 } & $\mathbf{S}$ & 93.59 & 0.010 & 93.57 & 93.60 \\
\cline { 2 - 6 } & $\mathbf{C}$ & 103.49 & 0.112 & 103.43 & 103.69 \\
\hline
\end{tabular}

the graph shows the relatively small difference caused by the simple ladder logic program modification compared with the larger difference caused by the complex modification.

Although the differences in mean execution times are discernible, some unexpected trends appear. Specifically, 19.015-S executes slower than 19.015-N, 16.056-S executes faster than 16.056-N and 19.011-S executes faster than 19.011-N. This is unexpected because, when an operation is added in the case of the simple modification, more work is created for the programmable logic controller, which should increase the mean execution time of the ladder logic program. The decrease in the mean execution time is probably caused by changes to the cache block alignment in memory. If the cache block alignment is improved by adding the operation, the increased number of cache hits could account for the decrease in the mean execution time. Further research is needed to determine the specific cause of this unexpected decrease in mean execution time.

- Firmware modification: The smallest observed difference caused by a firmware modification is the difference between 19.011-S and 19.015-S, which is $0.15 \mu \mathrm{s}$. Note that, since the standard deviation of 19.011-S is $0.007 \mu s$, the mean execution time of $19.015-\mathrm{S}$ is 21 standard deviations away from the mean execution time of 19.011-S. The mean execution time of $16.056-\mathrm{S}$ is $90.28 \mu \mathrm{s}$, which is 451 standard deviations away from the mean execution time of 19.011-S. In all cases, firmware modi- 


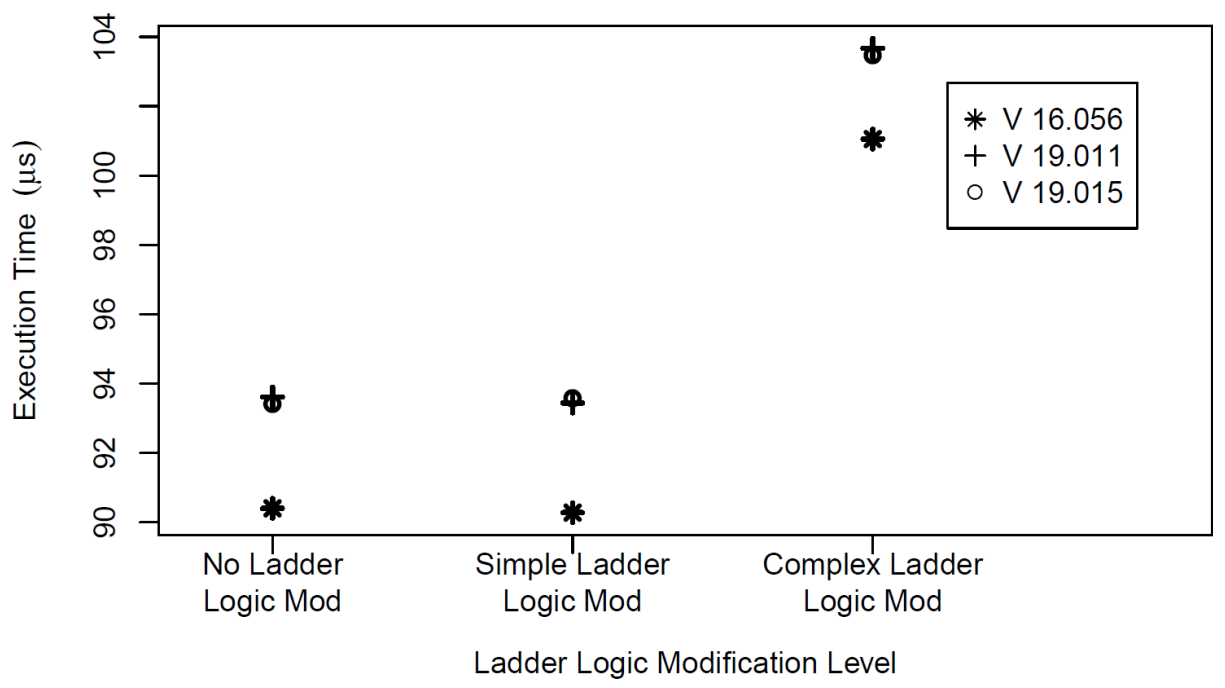

Figure 11: Mean execution times for Program 1.

fications create significant differences in the mean execution times that are readily discernible. Figure 11 shows the relatively small difference between firmware versions 19.011 and 19.015, compared with the large difference between firmware versions 19.011 and 16.056.

As in the case of the ladder logic program modifications, interesting trends are observed for firmware modifications. For all the ladder logic programs, firmware version 16.056 executes faster than the other two firmware versions. Because Program 1 contains a limited number of operations, the added complexity in the newer versions may outweigh any optimizations. Note that 19.015-N executes faster than 19.011-N and 19.015-C executes faster than 19.011-C. Because these two firmware versions have similar features, the complexity of the versions should be similar. Optimizations made in 19.015 may account for the decrease in the mean execution time. The decrease in the mean execution time due to the simple ladder logic program modification to version 19.011 (19.011-S) causes 19.011-S to execute faster than 19.015-S.

- Combined ladder logic program and firmware modification: It is interesting to examine the compound effects of modifying the ladder logic program and the firmware version. In most cases, the compound effect is discernible. In one case, however, the effect of modifying the ladder logic program is negated by the effect of modifying the 


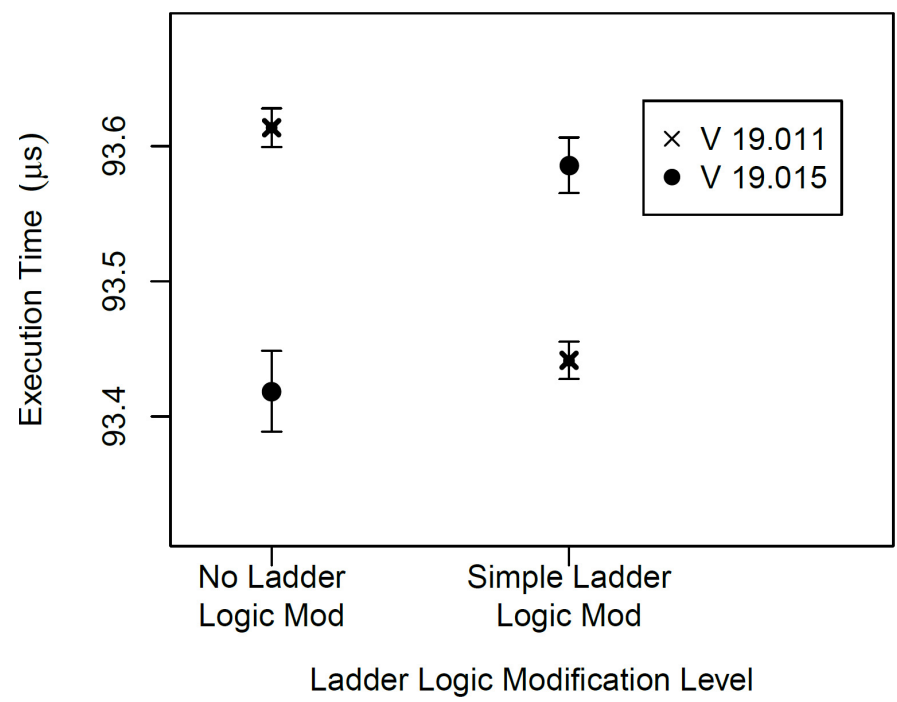

Figure 12: Compound effect with Program 1.

firmware version. The mean execution time of firmware version 19.015$\mathrm{N}$ is $0.19 \mu \mathrm{s}$ less than that of firmware version 19.011-N. The mean execution time of $19.015-\mathrm{S}$, however, is $0.17 \mu \mathrm{s}$ higher than $19.015-\mathrm{N}$. Figure 12 shows the mean execution times and 95\% confidence intervals for firmware versions 19.011-N, 19.011-S, 19.015-N and 19.015-S. Note that the confidence intervals overlap. In this case, the effect of the ladder logic program modification is negated by the effect of the firmware modification, yielding a total change of $0.02 \mu \mathrm{s}$ in the mean execution time. This change is not detected by the decision algorithm.

- Outlier: The standard deviation for the mean execution time of firmware version $19.015-\mathrm{C}$ is $0.112 \mu \mathrm{s}$. This standard deviation is significantly higher than the standard deviations obtained in the other experiments. Further investigation shows that the mean execution time for one repetition of $19.015-\mathrm{C}$ is $103.69 \mu \mathrm{s}$, which is significantly larger than the other four cases. This outlier negatively impacts the false positive rate of the experiment as explained in Section 5.4.1.

\subsubsection{Program 2 execution characteristics}

Table 5 presents the mean execution times for the five repetitions of Program 2. As in the case of Program 1, the mean, standard deviation, 
Table 5: Execution times ( $\mu s)$ for Program 2 (five repetitions).

\begin{tabular}{|c|c|c|c|c|c|}
\cline { 3 - 6 } \multicolumn{2}{c|}{} & Mean & SD & Min & Max \\
\hline \multirow{3}{*}{ V 16.056 } & N & $3,239.32$ & 0.341 & $3,238.78$ & $3,239.70$ \\
\cline { 2 - 6 } & $\mathbf{S}$ & $3,242.86$ & 0.192 & $3,242.72$ & $3,243.19$ \\
\cline { 2 - 6 } & $\mathbf{C}$ & $3,266.34$ & 0.140 & $3,266.15$ & $3,266.52$ \\
\hline & $\mathbf{N}$ & $3,182.20$ & 0.192 & $3,181.91$ & $3,182.43$ \\
\cline { 2 - 6 } V 19.011 & $\mathbf{S}$ & $3,221.77$ & 0.176 & $3,221.59$ & $3,222.01$ \\
\cline { 2 - 6 } & $\mathbf{C}$ & $3,230.74$ & 0.130 & $3,230.56$ & $3,230.89$ \\
\hline \multirow{3}{*}{$\mathbf{1 9 . 0 1 5}$} & $\mathbf{N}$ & $3,173.81$ & 0.123 & $3,173.66$ & $3,174.00$ \\
\cline { 2 - 6 } & $\mathbf{S}$ & $3,214.67$ & 0.125 & $3,214.55$ & $3,214.82$ \\
\cline { 2 - 6 } & $\mathbf{C}$ & $3,222.91$ & 0.150 & $3,222.64$ & $3,222.99$ \\
\hline
\end{tabular}

minimum and maximum are reported for each ladder logic program and firmware version combination. Note that $\mathrm{N}$ corresponds to no modification, $\mathrm{S}$ to simple modification and $\mathrm{C}$ to complex modification.

- Ladder logic program modification: For firmware version 16.056 with no modification to the ladder logic program (16.056-N), the mean execution time is $3,239.32 \mu \mathrm{s}$. With the simple modification (16.056-S), the mean execution time increases to $3,242.86 \mu \mathrm{s}$ - a difference of 3.54 $\mu s$. Note that, since the standard deviation of $16.056-\mathrm{N}$ is $0.341 \mu \mathrm{s}$, the mean execution time of 16.056-S is ten standard deviations away from the mean execution time of 16.056-N. The smallest observed difference in mean execution time caused by a ladder logic program modification is $3.54 \mu \mathrm{s}$. For firmware version 16.056 with the complex modification (16.056-C), the mean execution time is $3,266.34 \mu s$, which is 79 standard deviations away from the mean execution time of $16.056-\mathrm{N}$. The large difference in the mean execution times and the small standard deviations indicate that the modifications are readily identifiable.

Similarly, for firmware versions 19.011 and 19.015, modifications to the ladder logic program create significant differences in the mean execution times that are readily discernible. Figure 13 presents the mean execution times. The graph shows that the simple modification and complex modification cause the mean execution time to increase for all the firmware versions.

As in the case of Program 1, the differences in the mean execution 


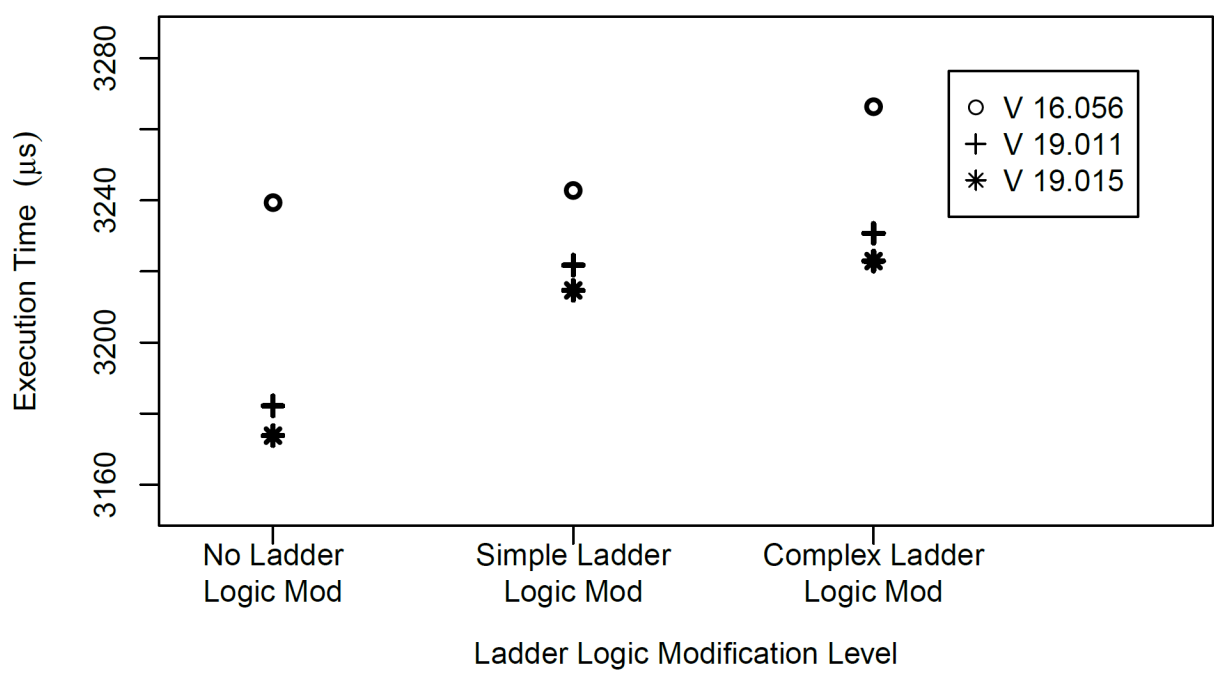

Figure 13: Mean execution times for Program 2.

times caused by ladder logic program modifications are discernible and interesting trends are observed. For firmware version 16.056, the simple modification increases the mean execution time by $3.54 \mu \mathrm{s}$. For firmware versions 19.011 and 19.015, however, the simple ladder logic program modification increases the mean execution time by approximately $40 \mu \mathrm{s}$. Similarly, the complex modification increases the mean execution time of firmware version 16.056 by $27 \mu \mathrm{s}$ whereas the complex modification increases the mean execution time of versions 19.011 and 19.015 by approximately $49 \mu \mathrm{s}$. The ladder logic program modifications have larger effects on the mean execution times with firmware versions 19.011 and 19.015 than with firmware version 16.056.

- Firmware modification: The smallest observed difference caused by a firmware modification is the difference between 19.011-S and 19.015-S, which is $7.10 \mu \mathrm{s}$. Note that, because the standard deviation of 19.011-S is $0.176 \mu \mathrm{s}$, the mean execution time of $19.015-\mathrm{S}$ is 40 standard deviations away from the mean execution time of 19.011-S. The mean execution time of $16.056-\mathrm{S}$ is $3242.86 \mu \mathrm{s}$, which is 120 standard deviations away from the mean execution time of 19.011-S.

In all cases, firmware modifications create significant differences in the mean execution times, which are readily discernible. Figure 13 shows the relatively small difference between firmware versions 19.011 and 
19.015 compared with the large difference between firmware versions 19.011 and 16.056. The graph also shows that the mean execution time decreases for the newer firmware versions; this is most likely due to optimizations in the newer versions. Regardless, all the firmware modifications are readily discernible.

- Combined ladder logic program and firmware modification: In the case of Program 2, there are no compound effects that result in similar mean execution times for two different ladder logic program and firmware combinations. Each ladder logic program and firmware combination yields a unique mean execution time.

- Outlier: As in the case of Program 1, one outlier is present in the Program 2 data. The standard deviation of the mean execution time of $16.056-\mathrm{N}$ is $0.341 \mu \mathrm{s}$. This standard deviation is significantly higher than the standard deviations obtained in the other experiments. The mean execution time for one repetition of $16.056-\mathrm{N}$ is $3,238.78 \mu \mathrm{s}$, which is significantly lower than the other four repetitions. Note that the outlier for Program 1 is 19.015-C whereas the outlier for Program 2 is 16.056-N. This outlier negatively impacts the false positive rate of the experiment as explained in Section 5.4.2.

\subsection{Decision algorithm}

The decision algorithm is designed to determine if a difference exists between two datasets and thus identify an anomaly. A permutation test is used to generate a p-value for the two datasets. A small p-value indicates that the two datasets are different. Since uncontrollable factors such as communications rates, external inputs and processor interrupts can occur, a .05 significance level is inadequate to make decisions about differences between two datasets. Instead, the p-value is used as a decision parameter with a decision threshold obtained by analyzing the results of the experiments. In practice, an industrial control operator would select a decision threshold based on the sensitivity of the system. For example, a nuclear power plant operator may decide that a higher false positive rate is acceptable to achieve a higher true positive rate. On the other hand, a toy factory operator may decide that the cost of a false positive error is more significant than the risk of a false negative error. In this study, the decision threshold was selected to optimize both the true positive rate and the false positive rate. 
The decision algorithm executes the following steps to determine if two datasets are different:

1. Two datasets are retrieved for comparison.

2. A permutation test is performed on the two datasets and the p-value of the test is obtained. A permutation test requires no distribution assumptions or special conditions [21].

3. A permutation test is performed on the two datasets and the p-value of the test is obtained. The decision algorithm in this study applies the one-way test in the Coin package in $\mathrm{R}$.

4. If the p-value is less than or equal to the decision threshold, then the datasets are considered to be different and a positive determination is reported.

5. If the p-value is greater than the decision threshold, then the datasets are considered to be not different and a negative determination is reported.

\subsection{Decision threshold selection}

This section discusses the procedure used to select the threshold for the decision algorithm. In practice, a control system operator would select a decision threshold based on the risk of a false negative error and the cost of a false positive error. A ROC curve is often used in machine learning to express the performance of a learning algorithm [10]. The process of building a ROC curve involves varying the decision threshold and recording the corresponding true positive and false positive rates. This process was used to generate the ROC curves for Program 1 and Program 2. The rates were then inspected to find an acceptable threshold.

Figures 14 and 15 present the ROC curves for Program 1 and Program 2, respectively. The area under the ROC curve for Program 1 is 0.96577 while the area under the ROC curve for Program 2 is 0.98333. Based on these two curves, a decision threshold of 0.0001 was selected because it provides an acceptable true positive rate and false positive rate for Program 1 and provides the lowest false positive rate for Program 2. This decision threshold was used for all determinations in the study.

\subsubsection{Modification detection rates}

The overall true positive rate is the rate at which the anomaly detection system detects modifications to the ladder logic program, firmware or both. 


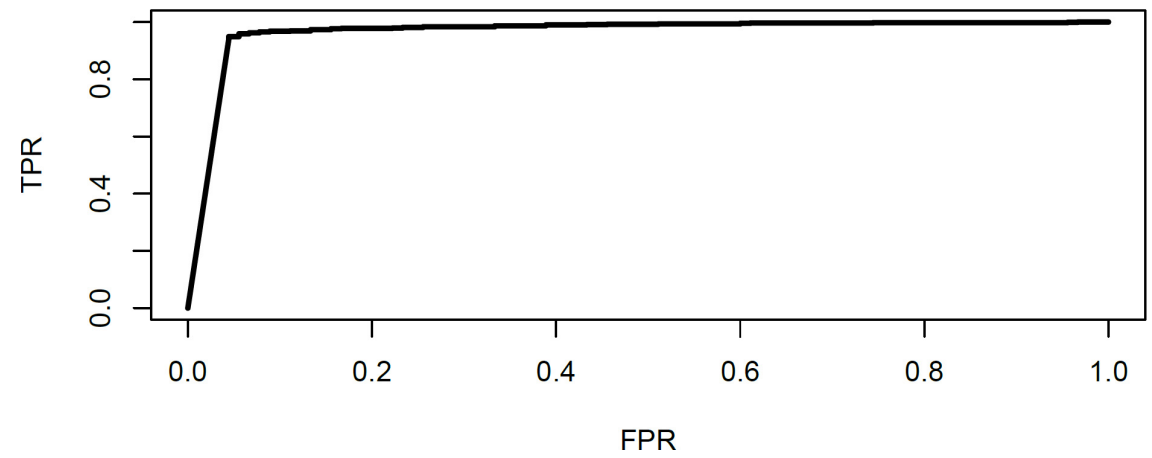

Figure 14: Program 1 ROC curve.

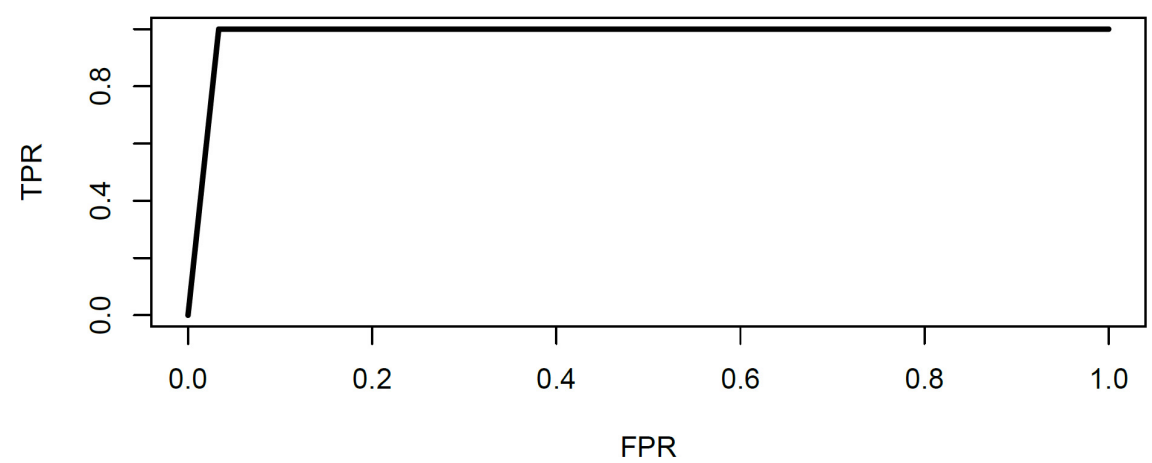

Figure 15: Program 2 ROC curve.

The ladder logic program true positive rate is the rate at which the anomaly detection system correctly identifies modifications to the ladder logic program when the firmware version is not modified. Similarly, the firmware true positive rate is the rate at which the anomaly detection system correctly identifies modifications to the firmware when the ladder logic program is not modified. The false positive rate is the rate at which the anomaly detection system incorrectly reports a modification when no modification was introduced.

\subsubsection{Program 1}

Table 6 shows the false positive and true positive rates of the anomaly detection system for Program 1. Note that the values in bold font correspond to false positive rates while all the other values correspond to true positive rates. Also, $\mathrm{N}$ corresponds to no modification, $\mathrm{S}$ to simple modification and $\mathrm{C}$ to complex modification. For all the comparisons, the overall true positive 
Table 6: Detection rates for Program 1 (threshold $=0.0001$ ).

\begin{tabular}{|c|c|c|c|c|c|c|c|c|c|c|}
\hline & \multicolumn{3}{|c|}{ V 16.056 } & \multicolumn{3}{|c|}{ V 19.011 } & \multicolumn{3}{|c|}{ V 19.015 } \\
\hline & & $\mathbf{N}$ & $\bar{S}$ & $\bar{C}$ & $\mathbf{N}$ & $\overline{\mathrm{S}}$ & $\bar{C}$ & $\overline{\mathbf{N}}$ & $\bar{S}$ & $\mathrm{C}$ \\
\hline \multirow{3}{*}{ V 16.056} & $\mathbf{N}$ & 0 & 1 & 1 & 1 & 1 & 1 & 1 & 1 & 1 \\
\hline & $\mathbf{S}$ & - & 0 & 1 & 1 & 1 & 1 & 1 & 1 & 1 \\
\hline & $\mathbf{C}$ & - & - & 0 & 1 & 1 & 1 & 1 & 1 & 1 \\
\hline \multirow{3}{*}{ V 19.011 } & $\mathbf{N}$ & - & - & - & 0 & 1 & 1 & 1 & 1 & 1 \\
\hline & $\mathbf{S}$ & - & - & - & - & $\overline{0}$ & 1 & 1 & 1 & 1 \\
\hline & $\mathbf{C}$ & - & - & - & - & - & 0 & 1 & 1 & 0.8 \\
\hline \multirow{3}{*}{ V 19.015} & $\mathbf{N}$ & - & - & - & - & - & - & $\overline{0}$ & $\frac{1}{1}$ & 1 \\
\hline & $\mathbf{S}$ & - & - & - & - & - & - & - & 0 & 1 \\
\hline & $\mathbf{C}$ & - & - & - & - & - & - & - & - & 0.4 \\
\hline
\end{tabular}

rate is 0.939 and the overall false positive rate is 0.044 . This means that the anomaly detection system correctly identifies modifications for $93.9 \%$ of the comparisons with a decision threshold of 0.0001 ; it incorrectly reports a difference $4.4 \%$ of the time.

Investigations revealed that the false positive rate is negatively impacted by the outlier discussed above. The false positive rate for 19.015 - $\mathrm{C}$ is 0.4 . This outlier is incorrectly identified as an anomaly when compared with the other four repetitions. No other false positives exist for Program 1. Additionally, the total true positive rate is negatively impacted due to the compound effect discussed in Section 4. The difference between 19.011-S and 19.015- $\mathrm{N}$ is not detected. Also, the difference between 19.011-N and 19.015-S is not detected. The true positive rate for these comparisons is zero.

The true positive rate for the ladder logic program modification (Program 1) is 1 . The anomaly detection system correctly identifies all the modifications made to Program 1.

The true positive rate for firmware modification with Program 1 is 0.98 . The true positive rate is negatively impacted by the comparisons between 19.011-C and 19.015-C, which contain the only incorrect determinations. The true positive rate for the comparisons between $19.011-\mathrm{C}$ and $19.015-\mathrm{C}$ is 0.8 . Further investigation revealed that the anomaly detection system incorrectly identifies the outlier from 19.015-C. The differences between this outlier and the samples of version 19.011-C are not detected; all the other firmware modifications are detected by the anomaly detection system. 
Table 7: Detection rates for Program 2 (threshold $=0.0001$ ).

\begin{tabular}{|c|c|c|c|c|c|c|c|c|c|c|}
\hline & \multicolumn{3}{|c|}{ V 16.056 } & \multicolumn{3}{|c|}{ V 19.011 } & \multicolumn{3}{|c|}{$\overline{V 19.015}$} \\
\hline & & $\mathbf{N}$ & $\mathbf{S}$ & $\bar{C}$ & $\mathbf{N}$ & $\overline{\mathbf{S}}$ & $\bar{C}$ & $\overline{\mathbf{N}}$ & $\mathbf{S}$ & $\bar{C}$ \\
\hline \multirow{3}{*}{ V 16.056} & $\mathbf{N}$ & 0.3 & 1 & 1 & 1 & 1 & 1 & 1 & 1 & 1 \\
\hline & $\mathbf{S}$ & - & $\overline{0}$ & $\frac{1}{1}$ & 1 & $\frac{1}{1}$ & $\frac{1}{1}$ & $\frac{1}{1}$ & $\frac{1}{1}$ & $\frac{1}{1}$ \\
\hline & $\mathrm{C}$ & - & - & 0 & 1 & 1 & 1 & 1 & 1 & 1 \\
\hline \multirow{3}{*}{ V 19.011 } & $\mathbf{N}$ & - & - & - & 0 & 1 & 1 & 1 & 1 & 1 \\
\hline & $\mathbf{S}$ & - & - & - & - & $\overline{0}$ & $\overline{1}$ & $\overline{1}$ & 1 & $\overline{1}$ \\
\hline & $\mathbf{C}$ & - & - & - & - & - & 0 & 1 & 1 & 1 \\
\hline \multirow{3}{*}{ V 19.015} & $\mathbf{N}$ & - & - & - & - & - & - & 0 & 1 & 1 \\
\hline & $\mathbf{S}$ & - & - & - & - & - & - & - & 0 & $\overline{1}$ \\
\hline & $\mathrm{C}$ & - & - & - & - & - & - & - & - & 0 \\
\hline
\end{tabular}

\subsubsection{Program 2}

Table 7 shows the true positive and false positive rates of the anomaly detection system for Program 2. Note that the values in bold font correspond to false positive rates while all the other values correspond to true positive rates. Also, $\mathrm{N}$ corresponds to no modification, $\mathrm{S}$ to simple modification and $\mathrm{C}$ to complex modification. The overall true positive rate is 1 and the overall false positive rate is 0.033 . This means that the anomaly detection system correctly identifies modifications for $100 \%$ of the comparisons with a decision threshold of 0.0001 ; it incorrectly reports a modification $3.3 \%$ of the time.

Investigations revealed that the false positive rate is negatively impacted by the outlier discussed above. The false positive rate for $16.056-\mathrm{N}$ is 0.3 . The anomaly detection system incorrectly identifies a difference when the outlier is compared with three of the four other repetitions.

The true positive rate for the ladder logic program modification (Program 2 ) is 1 . The anomaly detection system correctly identifies all the modifications made to Program 2.

The true positive rate for firmware modification with Program 2 is also 1. The anomaly detection system correctly identifies all the modifications made to the firmware with Program 2.

\subsection{Number of measurements}

While collecting more measurements is expected to increase the sensitivity of anomaly detection, it also takes more time. This section discusses the 
Table 8: Detection rates with varying number of measurements for Program 1.

\begin{tabular}{ccccc}
\hline Measurements & $\begin{array}{c}\text { Time } \\
(\mathbf{m i n})\end{array}$ & $\begin{array}{c}\text { Firmware } \\
\text { TPR }\end{array}$ & $\begin{array}{c}\text { Ladder Logic } \\
\text { TPR }\end{array}$ & $\begin{array}{c}\text { Total } \\
\text { FPR }\end{array}$ \\
\hline 10,000 & 42 & 0.97778 & 1 & 0.04444 \\
5,000 & 21 & 0.97778 & 0.99111 & 0.11111 \\
2,500 & 10 & 0.91556 & 0.92 & 0.17778 \\
1,250 & 5 & 0.74667 & 0.73778 & 0.14444 \\
250 & 1 & 0.66667 & 0.68889 & 0.04444 \\
\hline
\end{tabular}

Table 9: Detection rates with varying number of measurements for Program 2.

\begin{tabular}{ccccc}
\hline Measurements & $\begin{array}{c}\text { Time } \\
(\mathbf{m i n})\end{array}$ & $\begin{array}{c}\text { Firmware } \\
\text { TPR }\end{array}$ & $\begin{array}{c}\text { Ladder Logic } \\
\text { TPR }\end{array}$ & $\begin{array}{c}\text { Total } \\
\text { FPR }\end{array}$ \\
\hline 10,000 & 42 & 1 & 1 & 0.03333 \\
5,000 & 21 & 1 & 1 & 0.03333 \\
2,500 & 10 & 1 & 1 & 0.05556 \\
1,250 & 5 & 1 & 1 & 0.05556 \\
250 & 1 & 0.97333 & 0.88444 & 0.07778 \\
\hline
\end{tabular}

accuracy of anomaly detection when fewer than 10,000 measurements are collected. Estimates of the amount of time required to collect the measurements are based on a rate of four measurements per second.

\subsubsection{Program 1}

Table 8 presents the true positive and false positive rates of the anomaly detection system for varying numbers of measurements for Program 1. To obtain the true positive and false positive rates for $n$ measurements, the analysis process was repeated using only the first $n$ measurements from each dataset. All the other data points were disregarded during the analysis.

As expected, the true positive rate decreases as the number of measurements decreases. Acceptable true positive rates greater than 0.9 are achieved with as few as 2,500 measurements, but the false positive rate increases above an acceptable level of 0.1 for 5,000 measurements. Interestingly, acceptable false positive rates are achieved for 10,000 measurements and for 250 measurements. The highest false positive rate is obtained for 2,500 measurements. Thus, a rough bell curve is created. 


\subsubsection{Program 2}

Table 9 presents the true positive and false positive rates of the anomaly detection system for varying numbers of measurements for Program 2. As in the case of Program 1, the true positive rate decreases as the number of measurements decreases. The false positive rate, however, increases as the number of measurements decreases. An acceptable false positive rate is achieved for just 250 measurements. An acceptable firmware true positive rate is also achieved for 250 measurements, but the ladder logic program true positive rate decreases to 0.88 for 250 measurements. Acceptable true positive and false positive rates are achieved for as few as 1,250 measurements. With only 1,250 measurements, anomalies can be detected within five minutes of the modification.

\section{Conclusions}

The timing-based side channel analysis technique presented in this paper enables control system operators to detect modifications to the firmware and ladder logic programs of programmable logic controllers. A field device can be fingerprinted when it is first deployed to create a baseline fingerprint specific to the application. Subsequent fingerprints taken of the device are compared against the baseline to ensure that no modifications have been made. The technique may be used to detect intentional as well as unintentional modifications in programmable logic controllers and to alert control system operators about the anomalies.

Note that the views expressed in this paper are those of the authors and do not reflect the official policy or position of the U.S. Air Force, U.S. Army, U.S. Department of Defense or U.S. Government.

IMPORTANT NOTE TO IJCIP TYPESETTERS: I have checked and edited the references in this paper myself. Please DO NOT MODIFY the references - except to add hyperlinks. Please contact the Journal Manager Ms. Ramya Vasudevan if you have any questions.

Professor Sujeet Shenoi, Editor-in-Chief, IJCIP 


\section{References}

[1] O. Aciicmez, W. Schindler and C. Koc, Cache based remote timing attack on the AES, in Topics in Cryptology - CT-RSA 2007, M. Abe (Ed.), Springer-Verlag, Berlin Heidelberg, Germany, pp. 271-286, 2007.

[2] D. Agrawal, B. Archambeault, J. Rao and P. Rohatgi, The EM sidechannel(s), Proceedings of the Fourth International Workshop on Cryptographic Hardware and Embedded Systems, pp. 29-45, 2003.

[3] C. Arnold, J. Butts and K. Thirunarayan, Strategies for combating sophisticated attacks, Journal of Information Warfare, vol. 12(1), 2013.

[4] Z. Basnight, J. Butts, J. Lopez and T. Dube, Analysis of programmable logic controller firmware for threat assessment and forensic investigation, Journal of Information Warfare, vol. 12(2), 2013.

[5] D. Brumley and D. Boneh, Remote timing attacks are practical, Proceedings of the Twelfth USENIX Security Symposium, article no. 1, 2003.

[6] B. Chevallier-Mames, M. Ciet and M. Joye, Low-cost solutions for preventing simple side-channel analysis: Side-channel atomicity, IEEE Transactions on Computers, vol. 53(6), pp. 760-768, 2004.

[7] J. Dhem, F. Koeune, P. Leroux, P. Mestre, J. Quisquater and J. Willems, A practical implementation of the timing attack, in Smart Card Research and Applications, J. Quisquater and B. Schneier (Eds.), Springer-Verlag, Berlin Heidelberg, Germany, pp. 167-182, 2000.

[8] N. Falliere, L. O’Murchu and E. Chien, W32.Stuxnet Dossier, Version 1.4, Symantec, Mountain View, California, 2011.

[9] T. Hothorn, K. Hornik, M. van de Wiel and A. Zeileis, Implementing a class of permutation tests: The coin package, Journal of Statistical Software, vol. 28(8), 2008.

[10] N. Japkowicz and M. Shah, Evaluating Learning Algorithms: A Classification Perspective, Cambridge University Press, Cambridge, United Kingdom, 2011. 
[11] J. Kelsey, B. Schneier, D. Wagner and C. Hall, Side channel cryptanalysis of product ciphers, Journal of Computer Security, vol. 8(2-3), pp. $141-158,2000$.

[12] B. Kernighan and D. Ritchie, The C Programming Language, Prentice Hall, Englewood Cliffs, New Jersey, 1988.

[13] P. Kocher, Timing attacks on implementations of Diffie-Hellman, RSA, DSS and other systems, Proceedings of the Sixteenth International Conference on Advances in Cryptology, pp. 104-113, 1996.

[14] B. Kopf and D. Basin, An information-theoretic model for adaptive side-channel attacks, Proceedings of the Fourteenth ACM Conference on Computer and Communications Security, pp. 286-296, 2007.

[15] L. McMinn and J. Butts, A firmware verification tool for programmable logic controllers, in Critical Infrastructure Protection VI, J. Butts and S. Shenoi (Eds.), Springer, Heidelberg, Germany, pp. 59-69, 2012.

[16] T. Messerges, E. Dabbish and R. Sloan, Investigations of power analysis attacks on smartcards, Proceedings of the USENIX Workshop on Smartcard Technology, 1999.

[17] P. Neumann, Communication in industrial automation - What is going on? Control Engineering Practice, vol. 15(11), pp. 1332-1347, 2007.

[18] ODVA, Common Industrial Protocol (CIP), Ann Arbor, Michigan (www.odva.org/Technology-Standards/ Common-Industrial-Protocol-CIP/Overview), 2016.

[19] J. Pollet, Developing a solid SCADA security strategy, Proceedings of the Second ISA/IEEE Sensors for Industry Conference, pp. 148-156, 2002.

[20] R Foundation, The $\mathrm{R}$ Project for Statistical Computing (www. r-project.org), 2016.

[21] F. Ramsey and D. Schafer, The Statistical Sleuth: A Course in Methods of Data Analysis, Brooks Cole, Boston, Massachusetts, 2013. 
[22] H. Salem, A. Ternay and J. Smart, Brief history and use of chemical warfare agents in warfare and terrorism, in Chemical Warfare Agents: Chemistry, Pharmacology, Toxicology and Therapeutics, J. Romano, B. Lukey and H. Salem (Eds.), CRC Press, Boca Raton, Florida, pp. 1-20, 2008 .

[23] W. Schindler, A timing attack against RSA with the Chinese remainder theorem, Proceedings of the Second International Workshop on Cryptographic Hardware and Embedded Systems, pp. 109-124, 2000.

[24] J. Slay and M. Miller, Lessons learned from the Maroochy water breach, in Critical Infrastructure Protection, E. Goetz and S. Shenoi (Eds.), Springer, Boston, Massachusetts, pp. 73-82, 2008.

[25] D. Song, D. Wagner and X. Tian, Timing analysis of keystrokes and timing attacks on SSH, Proceedings of the Tenth USENIX Security Symposium, article no. 25, 2001.

[26] S. Stone and M. Temple, Radio-frequency-based anomaly detection for programmable logic controllers in the critical infrastructure, International Journal of Critical Infrastructure Protection, vol. 5(2), pp. 66-73, 2012 .

[27] K. Stouffer, J. Falco and K. Scarfone, Guide to Industrial Control Systems (ICS) Security, NIST Special Publication 800-82, National Institute of Standards and Technology, Gaithersburg, Maryland, 2011.

[28] J. Swets, Measuring the accuracy of diagnostic systems, Science, vol. 240(4857), pp. 1285-1293, 1988.

[29] G. Wilshusen, Cybersecurity: Threats Impacting the Nation, Testimony Before the Subcommittee on Oversight, Investigations and Management, Committee on Homeland Security, House of Representatives, GAO-12666T, United States Government Accountability Office, Washington, DC, 2012.

[30] R. Zabel, State of manufacturing and automation in the U.S. looks good, Automation.com, July 30, 2008.

[31] Y. Zhou and D. Feng, Side-Channel Attacks: Ten Years After its Publication and the Impacts on Cryptographic Module Security Testing, 
International Association for Cryptographic Research (www.iacr.org/ cryptodb/data/paper .php?pubkey=12722), 2005. 